\title{
High-Order Spectral Volume Method for 2D Euler Equations
}

\author{
Z.J. Wang (To receive correspondence) and Laiping Zhang \\ Department of Mechanical Engineering, Michigan State University \\ 2555 Engineering Building, East Lansing, MI 48824 \\ zjw@egr.msu.edu
}

Tel: 517-432-9132 Fax: 517-353-1750

Yen Liu

NASA Ames Research Center, Mail Stop T27B-1

Moffett Field, CA 94035

liu@nas.nasa.gov

Tel: 650-604-6667

\section{An extended abstract submitted to 16th AIAA CFD Meeting Orlando, Florida, June 23-26, 2003}

\begin{abstract}
The Spectral Volume (SV) method is extended to the 2D Euler equations. The focus of this paper is to study the performance of the SV method on multidimensional non-linear systems. Implementation details including total variation diminishing (TVD) and total variation bounded (TVB) limiters are presented. Solutions with both smooth features and discontinuities are utilized to demonstrate the overall capability of the SV method.
\end{abstract}

\section{Introduction}

We continue the development of the Spectral (Finite) Volume (SV) method for hyperbolic conservation laws on unstructured grids. Ultimately, the SV method is a Godunov-type finite volume method, which has been under development for several decades, and has become thestate-of-the-art for the numerical solution of hyperbolic conservation laws. Similar to the Godunov method, the SV method has two key components. One is data reconstruction, and the other is the (approximate) Riemann solver. What distinguishes the SV method from the k-exact finite volume (FV) method is in the data reconstruction. Instead of using a (large) stencil of neighboring cells to perform a high-order polynomial reconstruction, the unstructured grid cell called a spectral volume - is partitioned into a "structured" set of sub-cells called control volumes $(\mathrm{CVs})$, and cell-averages on these sub-cells are then the degrees-of-freedom (DOFs). These DOFs are used to perform a high-order polynomial reconstruction inside the SV. All the spectral volumes are partitioned in a geometrically similar manner in a simplex, and thus a single reconstruction is obtained. Next, the DOFs are updated to high-order accuracy using the usual Godunov method. Numerical tests with scalar conservation laws in both $1 D$ and $2 D$ and $1 D$ systems have verified that the SV method is indeed highly accurate, conservative, and geometrically flexible.

\section{Spectral Volume Method for the 2D Euler Equations}

The unsteady $2 \mathrm{D}$ Euler equation in conservative form can be written as 


$$
\frac{\partial Q}{\partial t}+\frac{\partial E}{\partial x}+\frac{\partial F}{\partial y}=0
$$

where $Q$ is the vector of conserved variables, $E$ and $F$ are the inviscid flux vectors. Assume that we solve (2.1) in the computational domain $\Omega$ subject to proper initial and boundary conditions. The domain $\Omega$ is discretized into $N$ non-overlapping triangular spectral volumes $(S V s)$

$$
\Omega=\bigcup_{i=1}^{N} S_{i} .
$$

Given a desired order of accuracy $k$ for (2.1), each spectral volume $S_{i}$ is then partitioned into $m=$ $k(k+1) / 2$ control volumes $(C V s)$, and the $j$-th $C V$ of $S_{i}$ is then denoted by $C_{i, j}$. Let $q$ denote any of the conservative variables in $Q$. The cell-averaged conservative variable $q$ at time $t$ for control volume $C_{i, j}$ is defined as

$$
\bar{q}_{i, j}(t)=\frac{\int_{C_{i, j}} q(x, y, t) d x d y}{V_{i, j}}, j=1, \ldots, m, i=1, \ldots, N,
$$

where $V_{i, j}$ is the volume of $C_{i, j}$. Given the cell-averaged conservative variables for all the $C V s$ in $S_{i}$, a polynomial $p_{i}(x, y) \in P^{k-1}$ (the space of polynomials of degree at most $k-1$ ) can be reconstructed such that it is a $k$-th order accurate approximation to the function $q(x, y)$ inside $S_{i}$ :

$$
p_{i}(x, y)=q(x, y)+O\left(h^{k}\right),(x, y) \in S_{i}, i=1, \ldots, N .
$$

This reconstruction can be solved analytically by satisfying the following conditions:

$$
\frac{\int_{C_{i, j}} p_{i}(x, y) d x d y}{V_{i, j}}=\bar{q}_{i, j}, j=1, \ldots, m .
$$

This polynomial $p_{i}(x, y)$ is the $k$-th order approximation we are looking for as long as the function $q(x, y)$ is smooth in the region covered by $S_{i}$. The reconstruction can be more conveniently expressed as

$$
p_{i}(x, y)=\sum_{j=1}^{m} L_{j}(x, y) \bar{q}_{i, j},
$$

where $L_{j}(x, y) \in P^{k-1}$ are the "shape" functions which satisfy

$$
\frac{\int_{C_{i, j}} L_{m}(x, y) d x d y}{V_{i, j}}=\delta_{j m} .
$$

The high-order reconstruction is then used to generate high-order updates for the cell-averaged state variable on the CVs. Integrating $(2.1)$ in $C_{i, j}$, we obtain the following integral equation for the $\mathrm{CV}$-averaged mean

$$
\frac{d \bar{Q}_{i, j}}{d t}+\frac{1}{V_{i, j}} \sum_{r=1}^{K} \int_{A_{r}}(f \bullet \boldsymbol{n}) d A=0
$$


where $\bar{Q}_{i, j}$ is the vector of the $C V$-averaged conservative variables in $C_{i, j}, f=(E, F), K$ is the number of faces in $C_{i, j}$, and $A_{r}$ represents the $r$-th face of $C_{i, j}$. The surface integration on each face can be performed with a $k$-th order accurate Gauss quadrature formula, i.e.

$$
\int_{A_{r}}(f \bullet \boldsymbol{n}) d A=\sum_{q=1}^{J} w_{r q} f\left(Q\left(x_{r q}, y_{r q}\right)\right) \bullet n_{r} A_{r}+O\left(A_{r} h^{k}\right),
$$

where $J=$ integer $[(k+1) / 2]$ is the number of quadrature points on the $r$-th face and, $w_{r q}$ are the Gauss quadrature weights, $\left(x_{r q}, y_{r q}\right)$ are the Gauss quadrature points, $h$ is the maximum edge length of all the CVs. Time $t$ is omitted whenever there is no confusion. If $f=$ constant, the following identity exists:

$$
\sum_{r=1}^{K} \int_{A_{r}}(f \bullet \boldsymbol{n}) d A=0 .
$$

Therefore, we will gain an extra order of accuracy if we sum up the surface integrals for the faces of $C_{i, j}$, i.e.,

$$
\sum_{r=1}^{K} \int_{A_{r}}(f \bullet n) d A=\sum_{r=1}^{K} \sum_{q=1}^{J} w_{r q} f\left(Q\left(x_{r q}, y_{r q}\right)\right) \bullet n_{r} A_{r}+O\left(A_{r} h^{k+1}\right) .
$$

Since $O\left(V_{i}\right)=O\left(A_{r} h\right)$, we therefore have

$$
\frac{1}{V_{i, j}} \sum_{r=1}^{K} \oint_{A_{r}}(f \bullet \boldsymbol{n}) d A=\frac{1}{V_{i, j}} \sum_{r=1}^{K} \sum_{q=1}^{J} w_{r q} f\left(Q\left(x_{r q,} y_{r q}\right)\right) \bullet n_{r} A_{r}+O\left(h^{k}\right) .
$$

With the polynomial distribution on each $S V$, the state variable is most likely discontinuous across the $S V$ boundaries. Therefore, the flux integration involves two discontinuous state variables just to the left and right of a face of the $S V$ boundary. This flux integration is carried out using an exact Riemann solver or one of the Lipschitz continuous approximate Riemann solvers or flux splitting procedures, i.e.,

$$
f\left(Q\left(x_{r q}, y_{r q}\right)\right) \bullet n_{r} \approx f_{R i e m}\left(Q_{L}\left(x_{r q}, y_{r q}\right), Q_{R}\left(x_{r q}, y_{r q}\right), \boldsymbol{n}_{r}\right),
$$

where $Q_{L}$ and $Q_{R}$ are the vector of conservative variables just to the left and right of a face. Substituting (2.12) into (2.11), we obtain

$$
\int_{A_{r}}(f \bullet \boldsymbol{n}) d A=\sum_{q=1}^{J} w_{r q} f_{\text {Riem }}\left(Q_{L}\left(x_{r q}, y_{r q}\right), Q_{R}\left(x_{r q}, y_{r q}\right), n_{r}\right) A_{r}+O\left(A_{r} h^{k}\right) .
$$

It is the Riemann solver which introduces the "upwinding", and dissipation into the SV method such that the SV method is not only high-order accurate, but also stable. In this paper, we employ Roe and local Lax-Friedrichs flux formula. Finally we obtain the following semi-discrete SV scheme

$$
\frac{d \bar{Q}_{i, j}}{d t}+\frac{1}{V_{i, j}} \sum_{r=1}^{K} \sum_{q=1}^{J} w_{r q} f_{R i e m}\left(Q_{L}\left(x_{r q}, y_{r q}\right), Q_{R}\left(x_{r q}, y_{r q}\right), n_{r}\right) A_{r}=0 .
$$

For time integration, we use the third-order TVD Runge-Kutta scheme from.

\section{Multi-Dimensional TVD and TVB Limiters}

For the non-linear Euler equations, it is necessary to perform data limiting to maintain stability if the solution contains discontinuities. There are two different ways of applying limiters in the system setting. One way is to apply a limiter to each characteristic variable. The other is to apply a limiter to each component of the vector of the conservative variables. The former has the nice 
property of naturally degenerating to the scalar case if the hyperbolic system is linear, but the latter is much more efficient. In this paper, we choose the component-wise approach because of its efficiency. To this end, we first establish the following numerical monotonicity criterion for each control volume

$$
\bar{q}_{i, j}^{\min } \leq q_{i, j}\left(x_{r q}, y_{y q}\right) \leq \bar{q}_{i, j}^{\max },
$$

where $\bar{q}_{i, j}^{\min }$ and $\bar{q}_{i, j}^{\max }$ are the minimum and maximum cell-averaged solutions among all its neighboring $C V s$ sharing a face with $C_{i, j}$, i.e.,

$$
\begin{aligned}
& \bar{q}_{i, j}^{\max }=\max \left(\bar{q}_{i, j}, \max _{1 \leq r \leq K} \bar{q}_{i, j, r}\right) \\
& \bar{q}_{i, j}^{\min }=\min \left(\bar{q}_{i, j}, \min _{1 \leq r \leq K} \bar{q}_{i, j, r}\right),
\end{aligned}
$$

and $q_{i, j}\left(x_{r q}, y_{y q}\right)$ is the reconstructed solution at any of the quadrature points. If (3.1) is strictly enforced, the resultant numerical scheme for the scalar case is TVD. However, it is well known that TVD schemes are locally first-order at extrema, and may pollute the global accuracy of the solution. In order to maintain the order of accuracy away from discontinuities, the TVB idea is followed here, i.e., small oscillations are allowed in the solution. If we express the reconstruction for the quadrature points in the following form

$$
\Delta q_{r q}=p_{i}\left(x_{r q}, y_{r q}\right)-\bar{q}_{i, j},
$$

then no data limiting is necessary if

$$
\left|\Delta q_{r q}\right| \leq 4 M_{q} h_{r q}^{2},
$$

where $h_{r q}=\left|r_{i, j}-r_{r q}\right|$ is the distance from the $\mathrm{CV}$ centroid to the quadrature point. In other words, no data limiting is necessary if (3.3) is satisfied, even if (3.1) is not. Usually the constant $M_{q}$ is chosen to be the maximum second derivative of the solution. However $M_{q}$ is a user chosen parameter if there is a discontinuity in the solution. Note that a different $M_{q}$ should be used for a different conservative variable. In this paper, $M_{q}$ is scaled according to the minimum and maximum of the component, i.e.,

$$
M_{q}=M\left(q_{\max }-q_{\min }\right) .
$$

where $\mathrm{M}$ is a constant independent of the component, and $q_{\max }$ and $q_{\min }$ are the maximum and minimum of the solution $q$ over the computational domain. If (3.3) is violated for any quadrature point, then it is assumed that the $\mathrm{CV}$ is close to a discontinuity, and the solution in the $\mathrm{CV}$ is locally linear, i.e.,

$$
q_{i, j}(x, y)=\bar{q}_{i, j}+\nabla q_{i, j} \bullet\left(r-r_{i, j}\right), \quad \forall \boldsymbol{r} \in C_{i, j} .
$$

The magnitude of the solution gradient is maximized subject to the monotonicity condition given in (3.1). The original high-order reconstruction in the CV is used to compute a initial guess of the gradient, i.e.,

$$
\nabla q_{i, j}=\left.\left(\frac{\partial p_{m}}{\partial x}, \frac{\partial p_{m}}{\partial y}\right)\right|_{r_{i, j}} .
$$

This gradient may not satisfy (3.1). Therefore it is limited by multiplying a scalar $\varphi \in[0,1]$ so that the following solution satisfy $(3.1)$

$$
q_{i, j}(x, y)=\bar{q}_{i, j}+\varphi \nabla q_{i, j} \bullet\left(r-r_{i, j}\right) .
$$

In the case of $M=0$, the limiter becomes TVD. The availability of cell-averaged data on the CVs inside a SV makes this CV-based data limiting possible, whereas in the $D G$ method, one can 
only do an element based data limiting. Due to the increased local resolution, the $S V$ method was shown to have better resolutions for discontinuities than the $D G$ method. This advantage should also carry over to the multi-dimensional systems case.

\section{Numerical Tests}

\section{Mach 3 Wind Tunnel with a Step}

The $2 \mathrm{D}$ wind tunnel is 3 units long and 1 unit wide, with a step of 0.2 units high located at 0.6 units from the tunnel inlet. The initial condition is a Mach 3 right-going uniform flow. Inviscid wall boundary conditions (reflective) are used for tunnel wall boundaries, while inflow and outflow boundary conditions are used at the inlet and exit of wind tunnel. It is well known that the corner of the step is a singularity, and can lead to a spurious Mach stem at the downstream bottom wall, and an erroneous entropy layer at the bottom wall. In the present study, no special treatments were used for the singularity to see how the singularity affects the numerical solutions.

Four different grids with various grid resolution near the singular comer point were employed. The first grid is nearly uniform with a mesh size of $h=0.025$. The other three grids are successively refined by halving the grid size near the corner. The four grids are shown in Figure 1. The computed density distributions at $t=4$ with the second order SV scheme and a TVB limiter $(M=10)$ is shown in Figure 2, and the corresponding third-order results are displayed in Figure 3. First it is evident that the spurious Mach stem weakened considerably with increased grid resolution near the corner point in both the second-order and third-order results. In addition, the entropy layer downstream the shock reflection point also weakened. Note that the third-order results have higher resolutions for the shock waves, as expected.

\section{Double Mach Reflection}

The computational domain for this problem is chosen to be $[0,4] \times[0,1]$. The reflecting wall lies at the bottom of the computational domain starting from $x=1 / 6$. Initially a right-moving Mach 10 shock is positioned at $x=1 / 6, y=0$ and makes a $60^{\circ}$ angle with the $x$-axis. For the bottom boundary, the exact post-shock condition is imposed for the region from $x=0$ to $x=1 / 6$ and a solid wall boundary condition is used for the rest. For the top boundary of the computational domain, the solution is set to describe the exact motion of the Mach 10 shock. The left boundary is set as the exact post-shock condition, while the right boundary is set as outflow boundary. The results at $t=0.2$ are shown. Figure 4 shows the density contours computed with the second SV scheme and a TVB limiter $(M=10)$ on the coarse $(\Delta x=\Delta y=1 / 60)$, medium $(\Delta x=\Delta y=1 / 120)$ and fine $(\Delta x=\Delta y=1 / 240)$ grids. The corresponding results for the third SV scheme are shown in Figure 5. It is obvious that the third order SV scheme captures the shock waves and the complex flow structure under the triple Mach stem better than the second-order SV scheme.

\section{Conclusions}

The Spectral Volume method has been successfully extended to two-dimensional hyperbolic systems of conservation laws. The extension to the system has been performed in a componentwise manner. Both TVD and TVB limiters are also implemented in a component-wise manner. This approach is very efficient, and has been shown to produce good numerical results for smooth and non-smooth flow problems. 

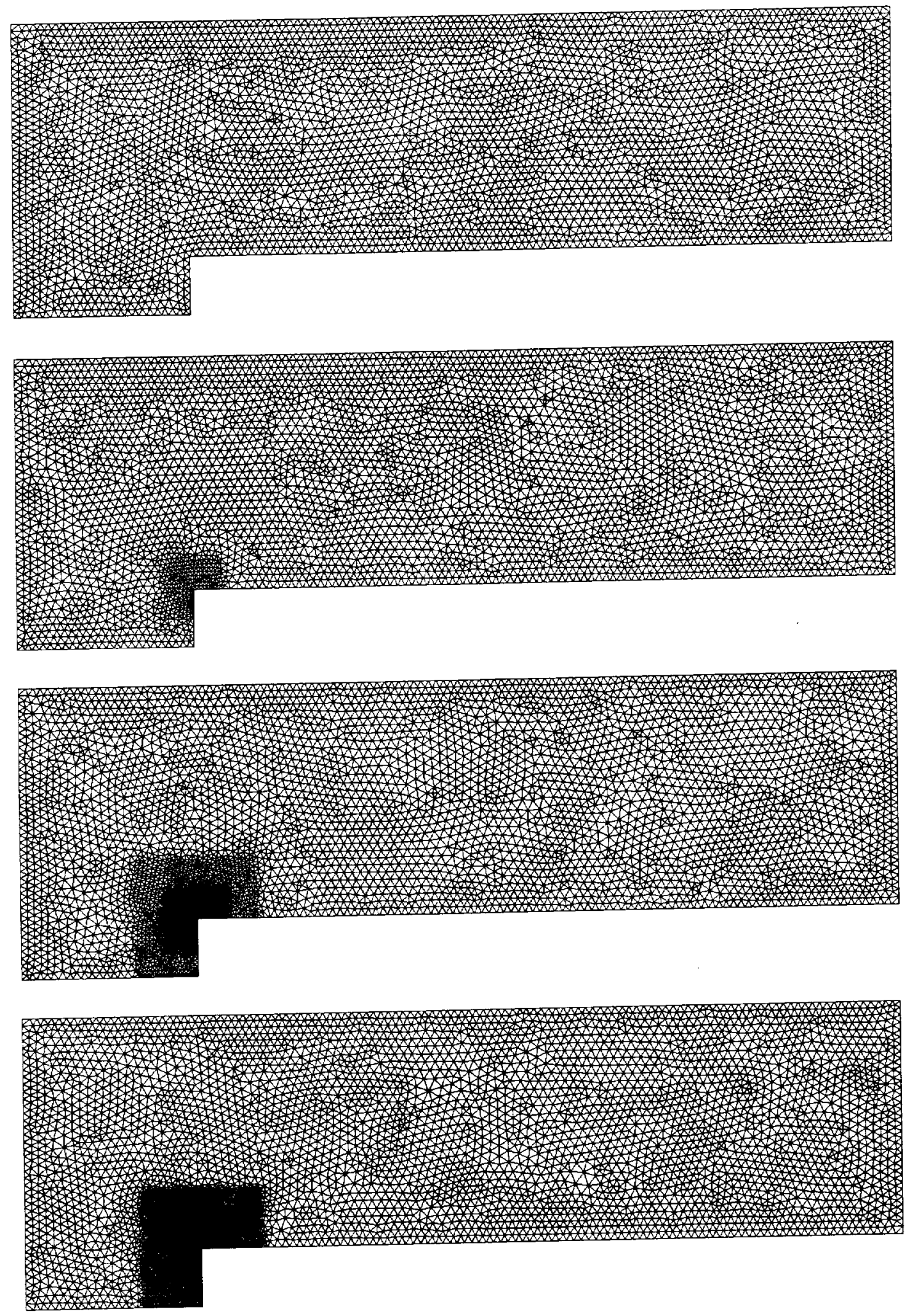

Figure 1. Four computational grids with different degrees of refinement near the singular corner point 

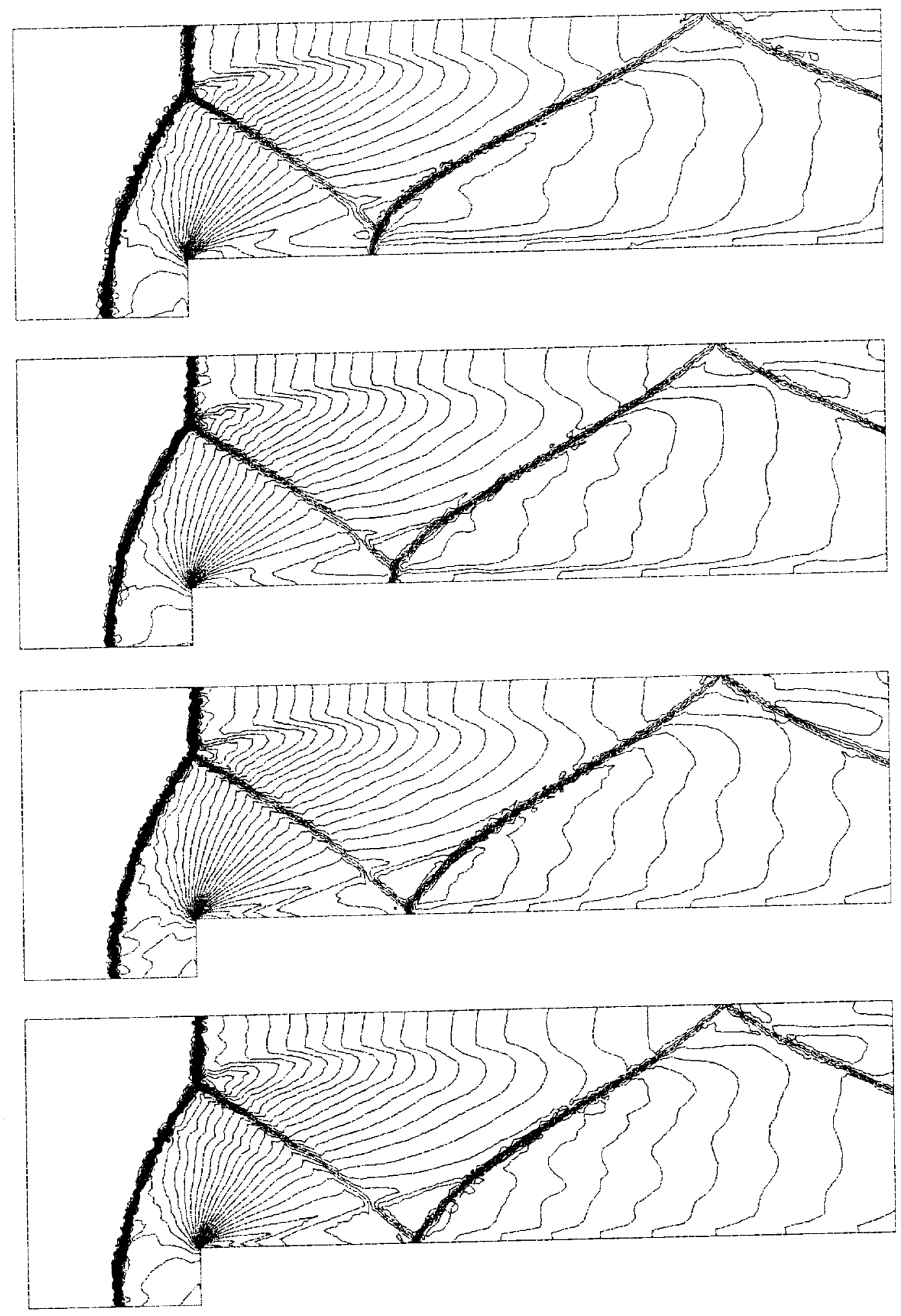

Figure 2. Computed density contours with a second-order SV scheme using a TVB limiter and $\mathrm{M}=10$ 

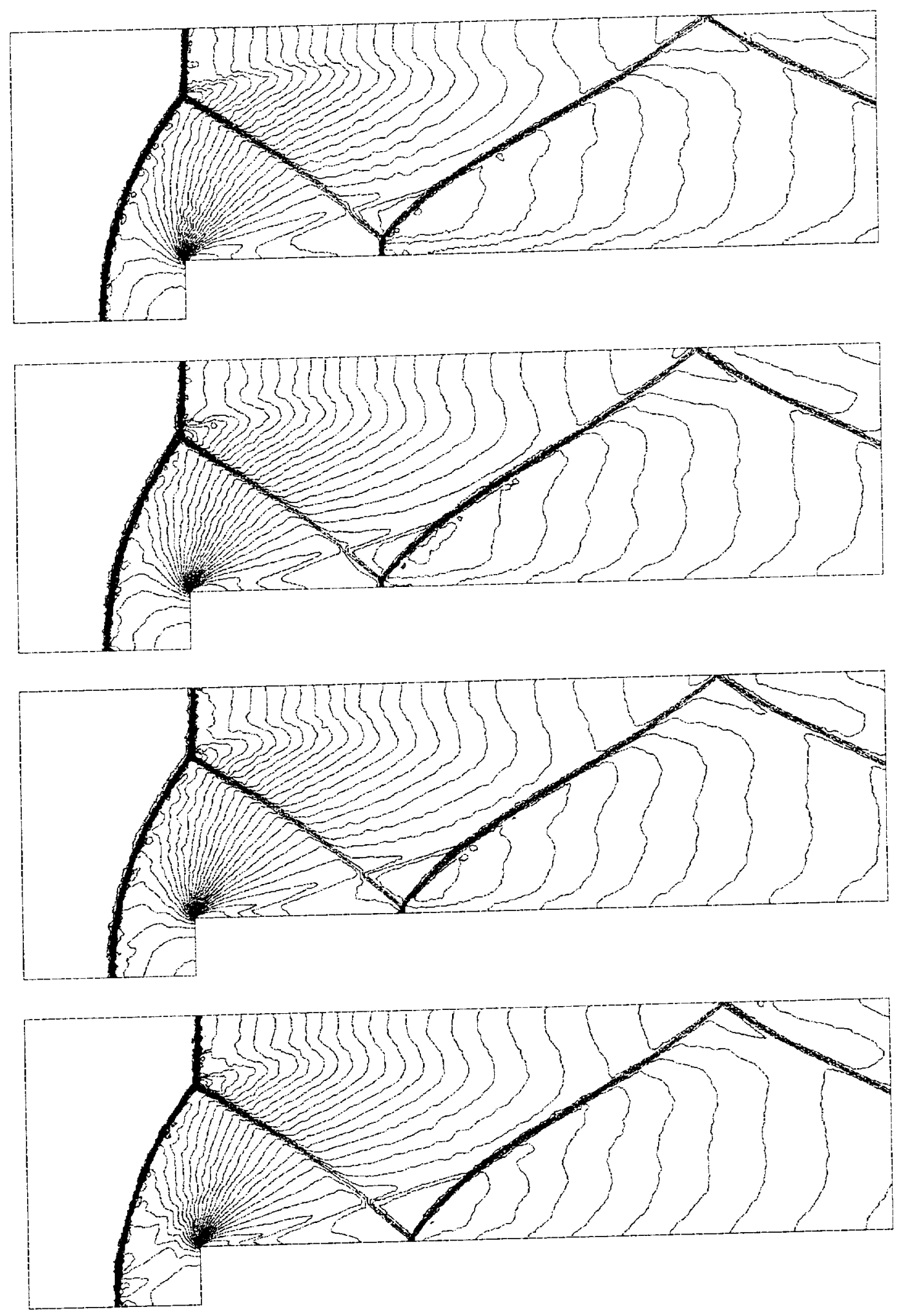

Figure 3. Computed density contours with a third-order SV scheme using a TVB limiter and $\mathrm{M}=10$ 


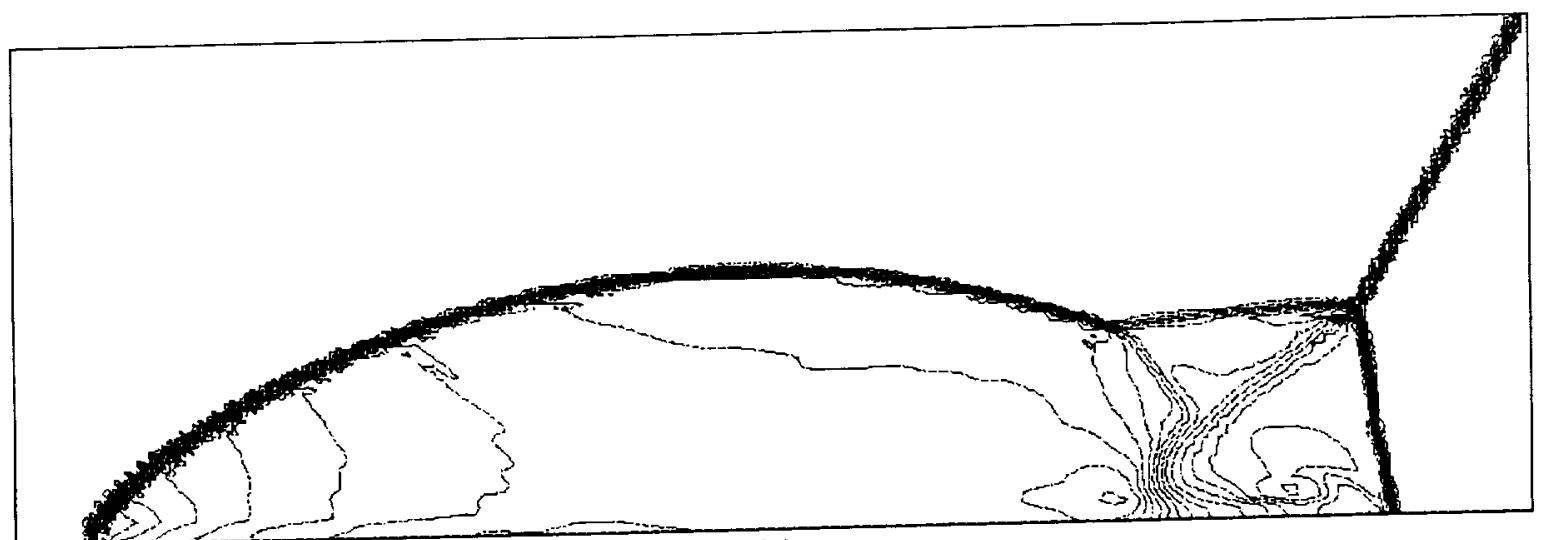

(a)

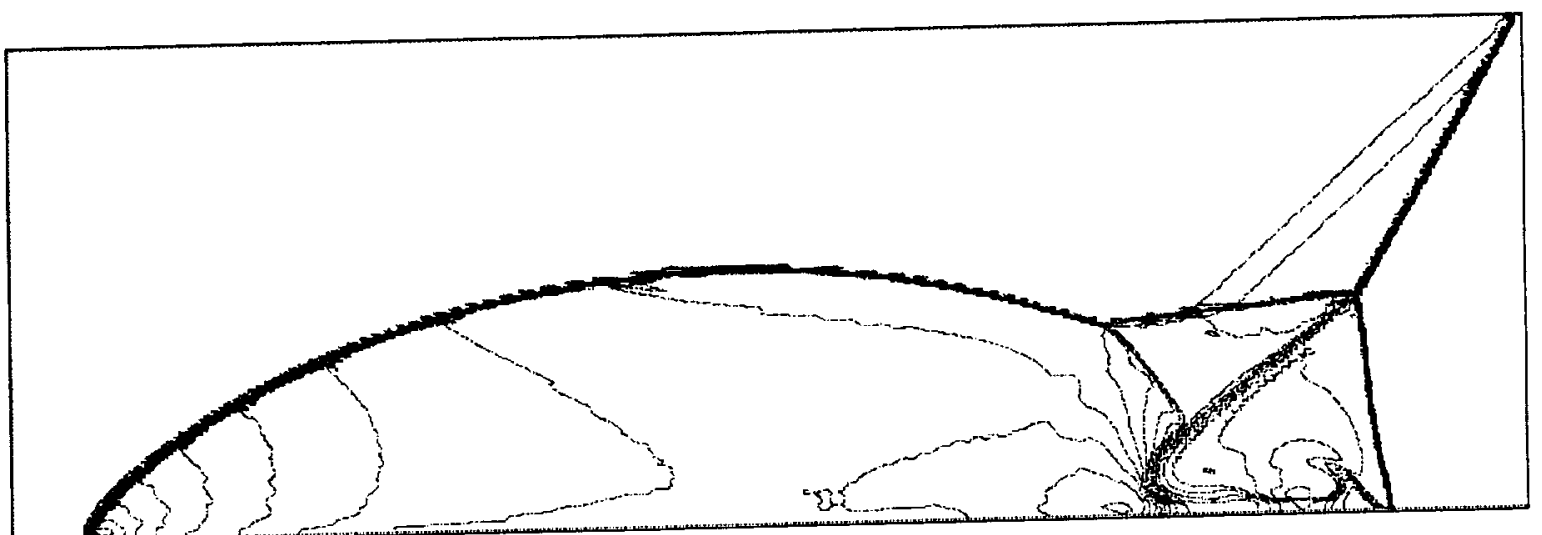

(b)

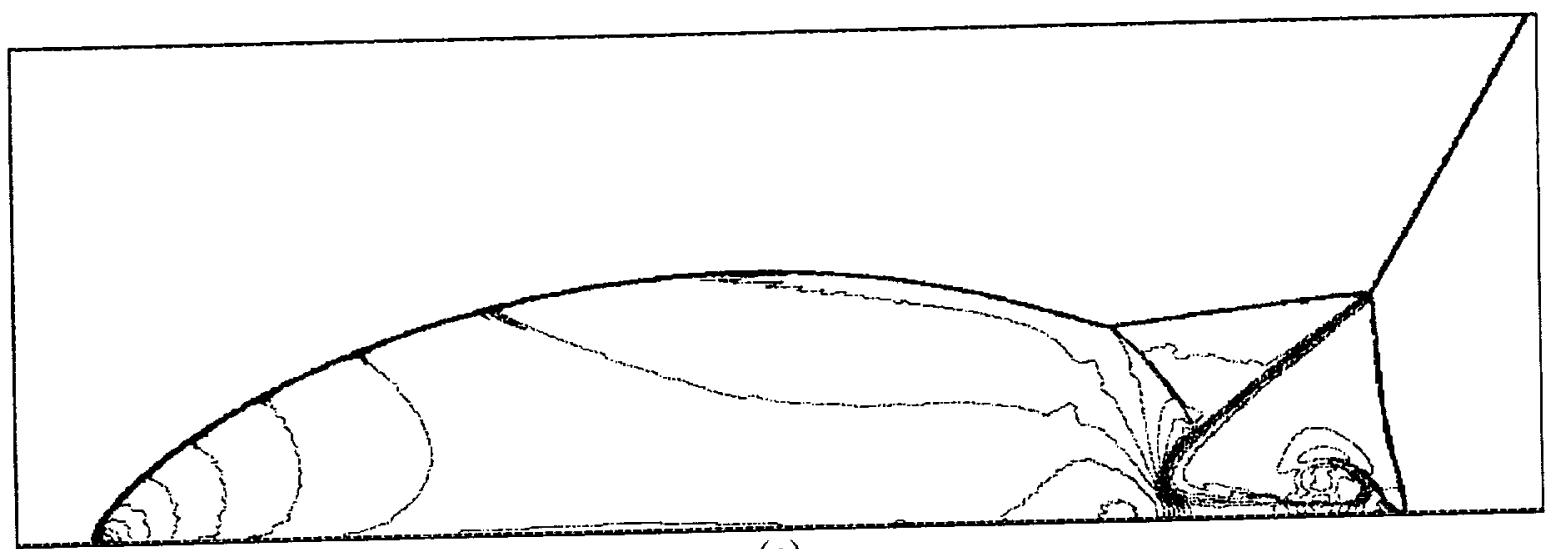

(c)

Figure 4. Density contours computed with the second order SV schemes and a TVB limiter $(\mathrm{M}=10)$ 


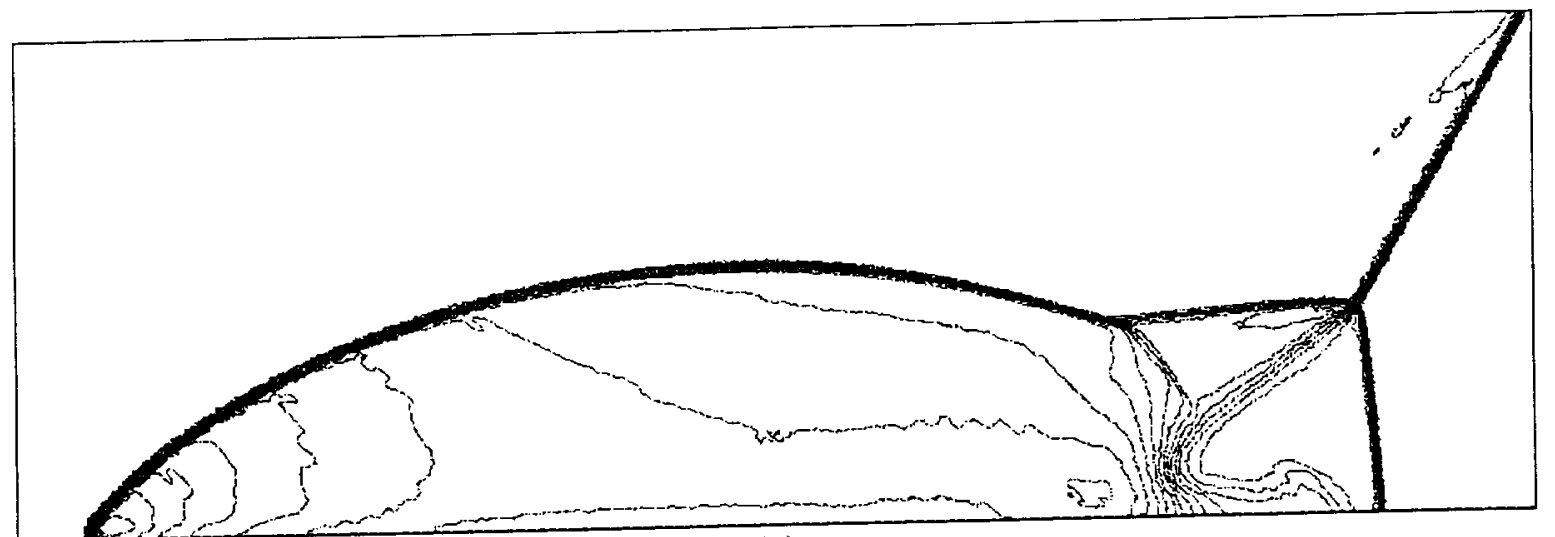

(a)

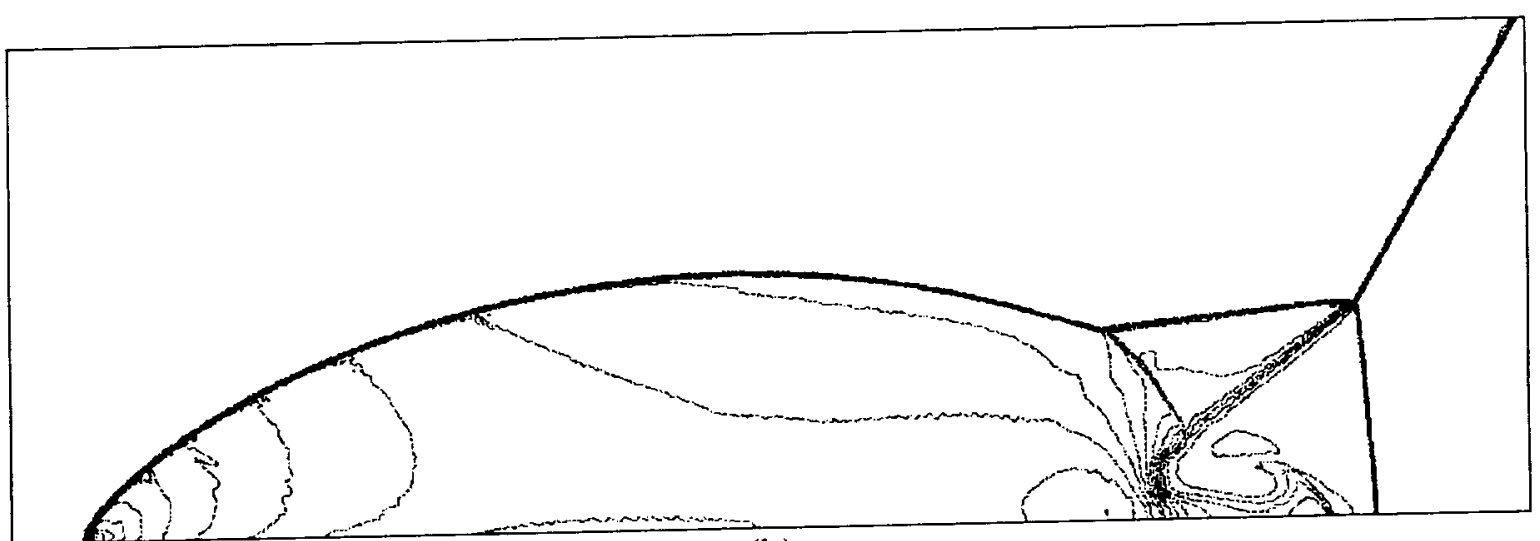

(b)

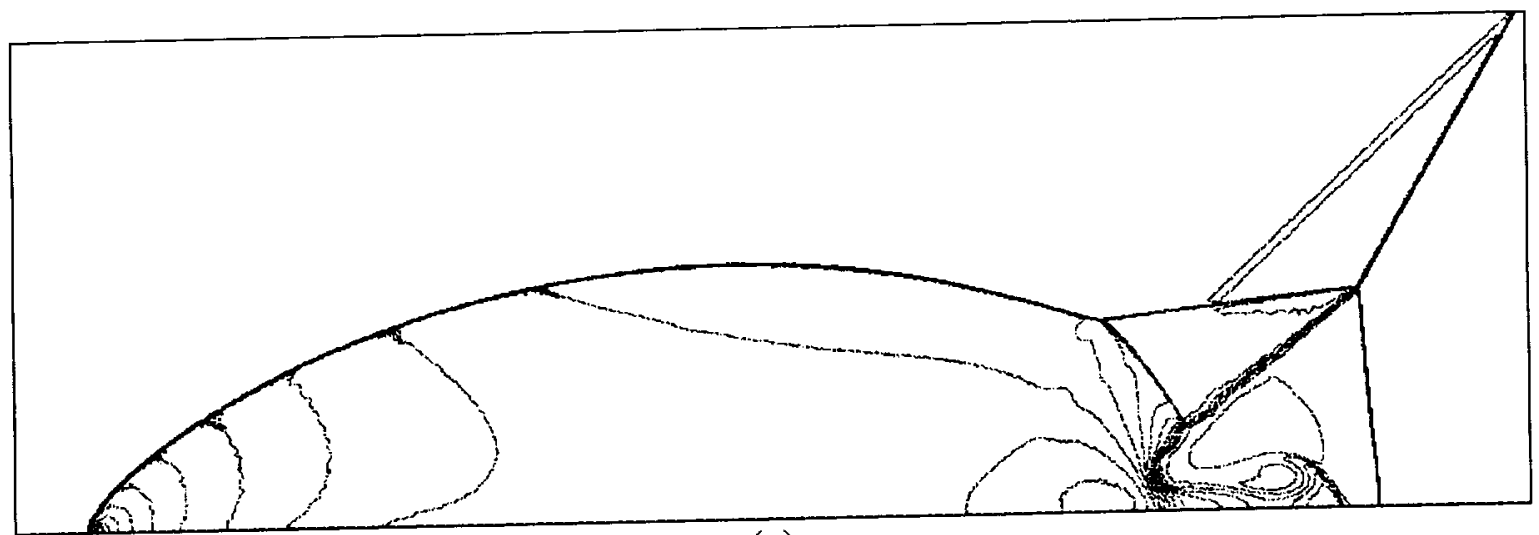

(c)

Figure 5. Density contours computed with the third order SV schemes and a TVB limiter $(\mathrm{M}=10)$ 